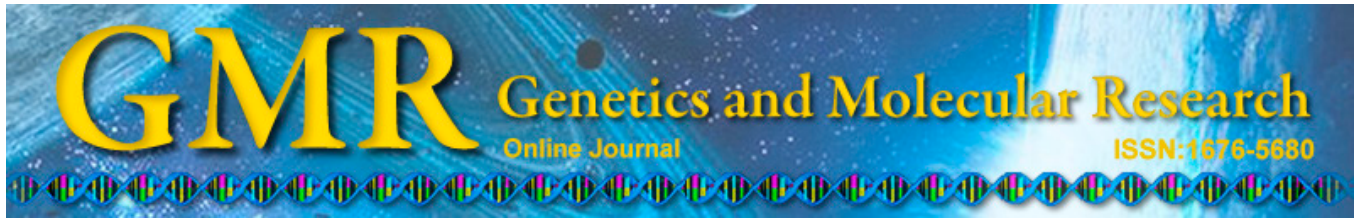

\title{
Association between the rs3795879 G/A polymorphism of the SERPINE2 gene and chronic obstructive pulmonary disease: a meta-analysis
}

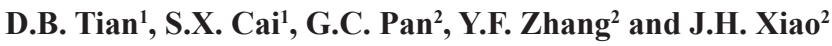 \\ ${ }^{1}$ Department of Respiratory and Critical Care Medicine, \\ Chronic Airway Diseases Laboratory, Nanfang Hospital, \\ Southern Medical University, Guangzhou, Guangdong, China \\ ${ }^{2}$ Qingyuan People's Hospital, Qingyuan, Guangdong, China \\ Corresponding author: S.X. Cai \\ E-mail: tiandongbo1111@163.com
}

Genet. Mol. Res. 14 (3): 7920-7928 (2015)

Received December 9, 2014

Accepted March 24, 2015

Published July 14, 2015

DOI http://dx.doi.org/10.4238/2015.July.14.18

\begin{abstract}
The present study aimed to investigate the effects of the rs3795879 polymorphism of the SERPINE2 gene on the development of chronic obstructive pulmonary disease (COPD) based on a systematic meta-analysis. An extensive literature search was performed to retrieve previously published case-control studies on the polymorphisms of SERPINE2 in COPD patients. Odds ratios (ORs) with $95 \%$ confidence intervals (CIs) were used to assess the association between SERPINE2 polymorphisms and risk of developing COPD. A total of 5 studies including 3034 COPD cases and 3068 controls were incorporated in the present meta-analysis. Generally, no significant association was identified between the rs 3795879 polymorphism of SERPINE2 and the risk of developing $\mathrm{COPD}(\mathrm{G}$ allele $v$ s A allele: $\mathrm{OR}=1.23,95 \% \mathrm{CI}=$ 0.97-1.32; $\mathrm{GG} v s \mathrm{GA}: \mathrm{OR}=1.19,95 \% \mathrm{CI}=0.81-1.76 ; \mathrm{GG} v s \mathrm{AA}: \mathrm{OR}=$
\end{abstract}


$1.23,95 \% \mathrm{CI}=0.89-1.70$; dominant model: $\mathrm{OR}=1.18,95 \% \mathrm{CI}=0.85$ 1.62; recessive model: $\mathrm{OR}=1.19,95 \% \mathrm{CI}=0.85-1.66$ ). In addition, subgroup analyses according to different ethnicities and the source of controls suggest no statistically significant association between the rs3795879 polymorphism of SERPINE2 and COPD risk. The results suggest that the rs 3795879 polymorphism of SERPINE2 is not a risk factor for COPD.

Key words: SERPINE2; rs3795879; SNP; COPD; Meta-analysis

\section{INTRODUCTION}

Chronic obstructive pulmonary disease (COPD) is a common inflammatory disease characterized by progressive airflow obstruction due to chronic bronchitis and emphysema (Ezzati and Lopez, 2003; Decramer et al., 2012). It has been reported that COPD is a multifactorial disease in which genetic and environmental factors are involved (Kabesch and Adcock, 2012). While environmental factors such as air pollution have been considered to be the main risk factor, genetic makeup of the hosts play an important role in the pathogenesis of COPD through regulating the expression of a number of proteins that are involved in the development of the disease (Chen et al., 2008; Haq et al., 2010).

The SERPINE2 gene that encodes the serine protease inhibitor E2, a member of the SERPIN family, is located on chromosome 2q33-35 (Carter et al., 1995). Recently, Demeo et al. (2006) have demonstrated that SERPINE2 is a novel risk factor for COPD. Zhu et al. (2007) further provided evidence supporting the association between SERPINE2 genetic polymorphisms and COPD based on two large population-based studies conducted in Bergen, Norway. However, the study by Zhong et al. (2009) reported that no significant association was found between five SERPINE2 genetic polymorphisms and COPD in a Han population in Southwest China. In addition, no significant association between single nucleotide polymorphisms (SNPs) in SERPINE2 and COPD was found in a prospective study including 1018 COPD cases and 911 controls in six European centers (Chappell et al., 2006). Considering the differences in the genetic backgrounds of the distinct ethnicities included in these studies ( $\mathrm{Li}$ et al., 2008), it is important to systematically analyze the data from these studies to identify possible genetic predispositions of SERPINE2 to COPD. Among the above-mentioned studies, the rs 3795879 polymorphism of SERPINE2 has been the best studied (Zhong et al., 2009; Fujimoto et al., 2010; Kukkonen et al., 2011; Wang et al., 2011). Therefore, the present meta-analysis was conducted to comprehensively analyze the potential association between the rs 3795879 polymorphism of SERPINE2 and the susceptibility to COPD.

\section{MATERIAL AND METHODS}

\section{Selection of published studies}

A systematic literature search was conducted in PubMed, Embase databases, and Web of Science (last publication date: October 15, 2014) using the following MeSH terms and key words: ("SNP" or "mutation" or "genetic polymorphism" or "variation" or "polymorphism" 
or "single nucleotide polymorphism" or "variant") and ("chronic obstructive pulmonary disease" or "COPD" or "chronic obstructive airway disease" or "chronic obstructive lung disease" or "chronic airflow obstruction") and ("serine protease inhibitor E2" or "SERPINE2"). The reference lists of retrieved studies were also searched to identify related studies. Studies included in the present meta-analysis met the following criteria: i) they investigated the association between rs3795879 polymorphisms and COPD risk, ii) they were case-controlled studies, iii) they provided available allele or genotype frequency for computing odds ratios (ORs) with 95\% confidence intervals (CIs), iv) we had access to full-text articles, and v) the distribution of the genotypes in the control group was in Hardy-Weinberg equilibrium (HWE). Major reasons for exclusion of a particular study were i) it was only a case-population study, ii) it was a study in which comparison outcomes were not reported or not possible to be determined, and iii) it was a duplicate of a previous study.

\section{Data extraction}

Two independent investigators reviewed the titles, abstracts, and full texts of retrieved articles independently using a standardized extraction form. For conflicting evaluations, an agreement was reached following consensus and discussion. For each study, the following characteristics were collected: first author's name, year of publication, country of origin, ethnicity, genotyping methods, COPD definition, mean age, smoking status, source of controls (population-based or hospital-based controls), total number of cases and controls, and the genotype distributions in cases and controls. No minimum number of patients was defined for this present meta-analysis.

\section{Statistical analyses}

All statistical analyses were performed using the STATA software (version 11.0; Stata Corporation, College Station, TX, USA), and all tests were two-sided with a significance level of 0.05 , unless stated otherwise. HWE in controls was assessed by the chi-squared test and a $\mathrm{P}$ value less than 0.05 was considered as significant disequilibrium. When HWE disequilibrium existed $(\mathrm{P}<0.05)$, or it was impossible to evaluate this equilibrium, sensitivity analysis was performed. The strength of the association between rs3795879 polymorphisms and COPD risk was evaluated based on ORs with $95 \%$ CIs. The chi-square-based Q statistic was used to test for heterogeneity among the studies included in the meta-analysis (Higgins et al., 2003). When the Q-test P value was no less than 0.1 , a fixed-effect model with Mantel-Haenszel method was used to calculate the pooled ORs (Petitti, 2000). Otherwise, a random-effect model with inverse variance method was used. The risk of COPD associated with rs3795879 polymorphisms was determined from a combination of single studies by heterozygote comparison (GA vs AA), homozygote comparison (GG vs AA), dominant models (GG + GA vs AA), recessive model (GG vs GA+AA), and allelic comparison (G vs A). In addition, we performed stratification analyses based on different ethnicities and the source of controls. One-way sensitivity analysis was performed to assess the stability of the results. Briefly, a single study included in the meta-analysis was removed each time to evaluate the influence of the individual data set to the pooled OR. Begg and Mazumdar (1994) adjusted rank correlation test and the Egger regression asymmetry test (Egger et al., 1997) were plotted to evaluate publication bias. 


\section{RESULTS}

\section{Characteristics of the studies}

Twenty-three articles that have been published by October 15, 2014 were identified by searching the PubMed, Embase, and Web of Science databases (Figure 1). By reviewing the abstracts and full texts, a total of 5 case-control studies that include 3034 cases and 3068 controls were finally included in this meta-analysis (Chappell et al., 2006; Demeo et al., 2006; Zhu et al., 2007; Zhong et al., 2009; Wang et al., 2011). Table 1 shows the characteristics of these five studies. The distribution of genotypes in the controls of all studies was consistent with the HWE.

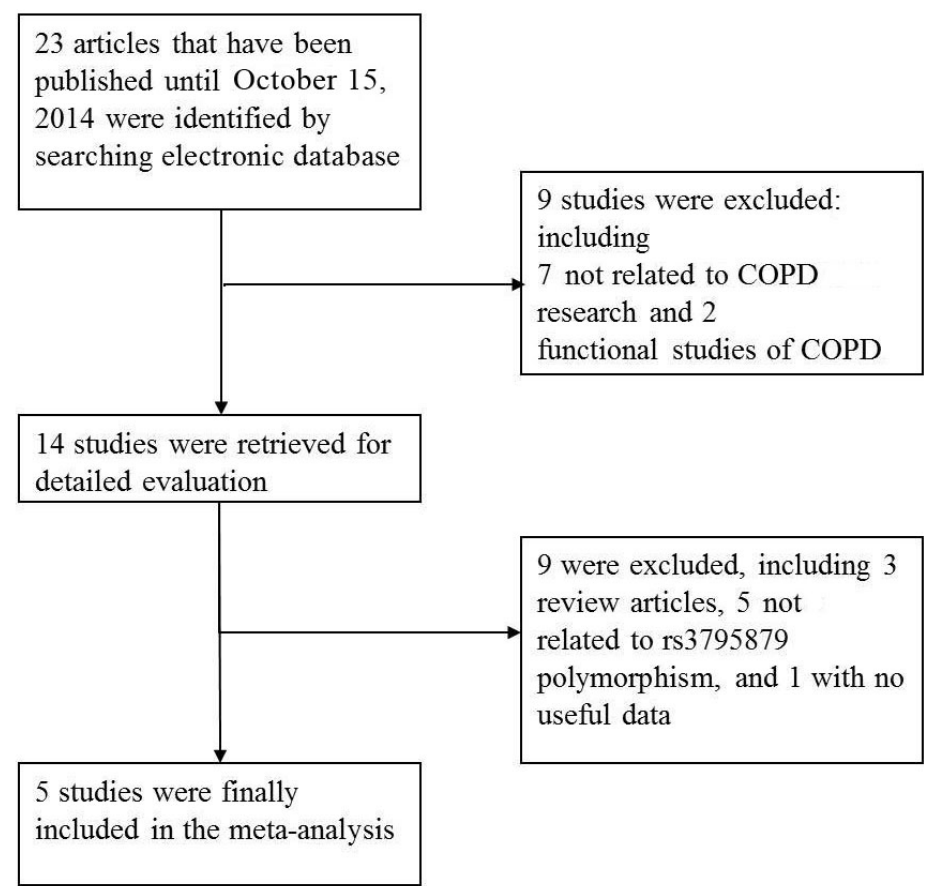

Figure 1. Studies included in this meta-analysis based on the criteria for inclusion and exclusion (see Material and Methods section).

Table 1. Pooled ORs and $95 \%$ CIs of stratified meta-analysis.

\begin{tabular}{|c|c|c|c|c|c|c|c|c|c|c|}
\hline First author & Country & Ethnicity & $\begin{array}{l}\text { Genotyping } \\
\text { method }\end{array}$ & COPD definition & $\begin{array}{l}\text { Mean age (years) } \\
\text { (case/control) }\end{array}$ & $\begin{array}{c}\text { Smoking } \\
\text { (case/control) }\end{array}$ & $\begin{array}{c}\text { Source of } \\
\text { control }\end{array}$ & Case & Control & $\begin{array}{l}\text { P of } \\
\text { HWE }\end{array}$ \\
\hline Wang (2011) & China & Asian & PCR-RFLP & FEV1/FVC $<70 \%$ & $65.1 / 63.7$ & Mixed/Mixed & HB & 409 & 411 & 0.34 \\
\hline Zhong (2009) & China & Asian & Sequencing & $\begin{array}{l}\text { Predicted FEV1 }<80 \% \\
\text { and FEV1/FVC }<70 \%\end{array}$ & $71.4 / 67.0$ & Yes/Yes & HB & 327 & 349 & 0.57 \\
\hline Zhu (2007) & Norway & Caucasian & $\mathrm{ABI}$ sequencer & $\begin{array}{l}\text { Predicted FEV1 }<60 \% \\
\text { and FEV1/FVC }<90 \%\end{array}$ & $65.5 / 55.5$ & Mixed/Mixed & PB & 973 & 956 & $>0.05$ \\
\hline Demeo (2006) & USA & Caucasian & Taqman & Predicted FEV $1<45 \%$ & NR & NR & HB & 243 & 441 & 0.18 \\
\hline Chappell (2006) & UK & Caucasian & Taqman & NR & NR & NR & PB & 1018 & 911 & 0.55 \\
\hline
\end{tabular}

$\mathrm{NR}=$ not reported; $\mathrm{HB}=$ hospital-based control; $\mathrm{PB}=$ population-based control. 


\section{Meta-analysis}

The association between rs3795879 polymorphisms and the risk of developing COPD is presented in Table 2. Overall, no significant association between rs 3795879 polymorphisms and the risk of developing COPD was identified based on all genetic models of rs3795879 polymorphisms (G allele $v s$ A allele: $\mathrm{OR}=1.23,95 \% \mathrm{CI}=0.97-1.32$; $\mathrm{GG} v s \mathrm{GA}: \mathrm{OR}=1.19,95 \% \mathrm{CI}$ $=0.81-1.76 ;$ GG $v s$ AA: $\mathrm{OR}=1.23,95 \% \mathrm{CI}=0.89-1.70 ;$ dominant model: $\mathrm{OR}=1.18,95 \% \mathrm{CI}=$ 0.85-1.62; recessive model: $\mathrm{OR}=1.19,95 \% \mathrm{CI}=0.85-1.66$ ) (Figure 2). In addition, no statistically significant association was identified between the SERPINE2 rs3795879 polymorphism and COPD risk based on analyses of a stratified population (Table 2).

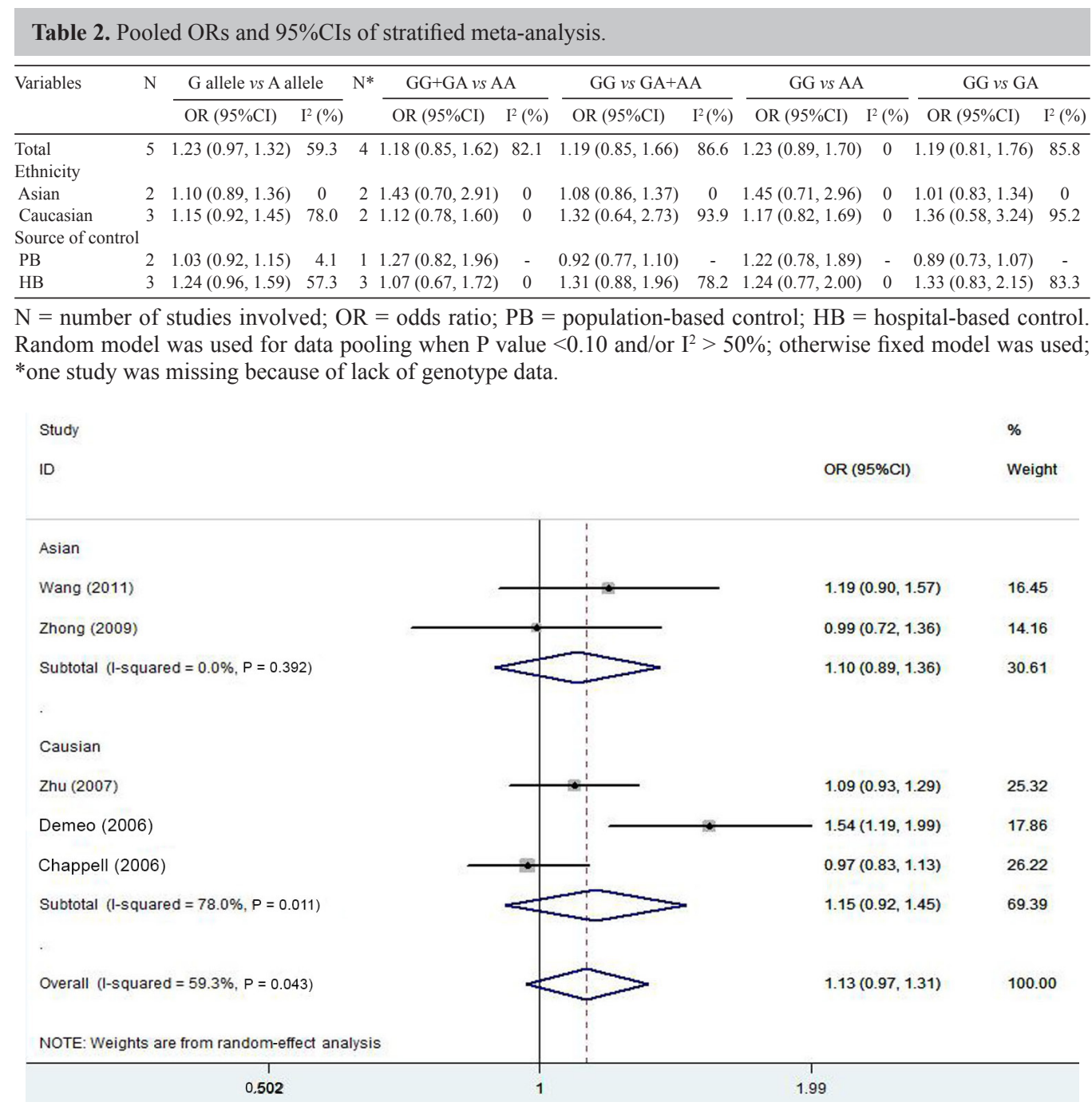

Figure 2. Forest plot of odds ratios (ORs) of the rs 3795879 polymorphism (G allele $v s$ A allele) associated with COPD stratified by ethnicity. 


\section{Test of heterogeneity}

Significant heterogeneity was identified between all studies in the $\mathrm{G} v s$ A comparison of the rs 3795879 polymorphism $\left(I^{2}=59.3 \%, \mathrm{P}=0.04\right)$. When stratified by ethnicity, no heterogeneity was observed between the studies conducted with Asians.

\section{Sensitivity analyses and publication bias evaluation}

No significant deviation from HWE was detected by examining the genotype frequencies in the controls, suggesting that the final results of this meta-analysis were relatively stable and reliable. Both Begg and Egger tests were conducted to assess the publication bias. No evidence of publication bias was identified in all comparison models in this meta-analysis (Table 3 and Figure 3).

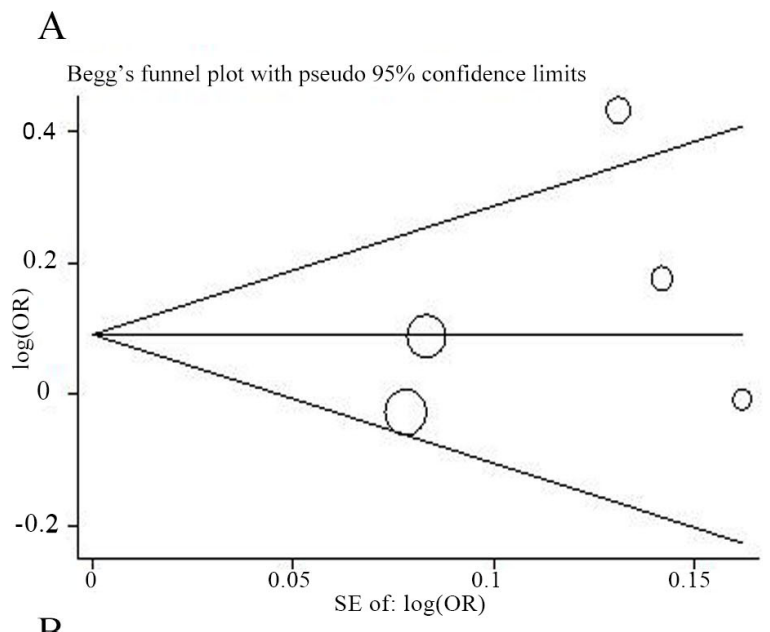

B

Egger's publication bias plot

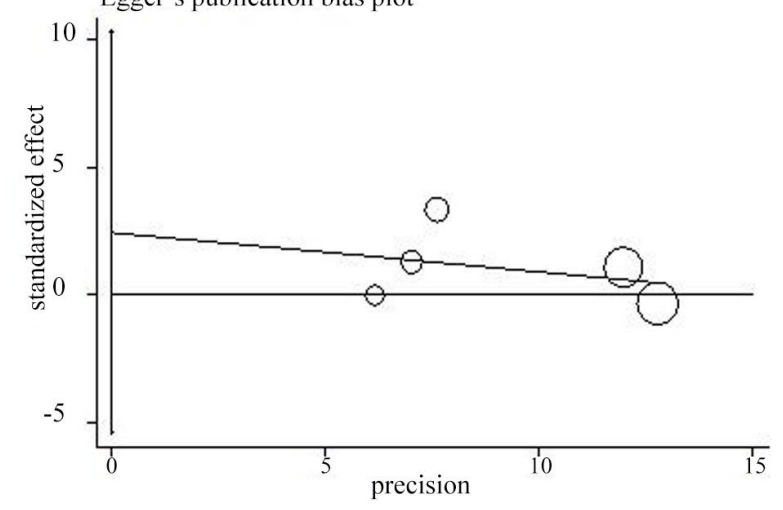

Figure 3. Begg's and Egger's funnel plots for publication bias test. No significant publication bias was found. Each point represents a separate study for the indicated association. $\log (\mathrm{OR})$, natural logarithm of OR. Horizontal line, mean effect size. 
Table 3. Results of Begg and Egger tests for the evaluation of publication bias.

\begin{tabular}{lcccc}
\hline Comparison type & \multicolumn{2}{c}{ Begg test } & \multicolumn{2}{c}{ Egger test } \\
\cline { 2 - 3 } \cline { 5 - 5 } & $z$ & $\mathrm{P}$ & $t$ & $\mathrm{P}$ \\
\hline G/A & 0.24 & 0.806 & 0.99 & 0.396 \\
GG/GA & 0.34 & 0.734 & 1.03 & 0.412 \\
GG/AA & -0.34 & 1.000 & 0.41 & 0.721 \\
Dominant model & -0.34 & 1.000 & 0.06 & 0.961 \\
Recessive model & -0.34 & 1.000 & 0.97 & 0.434 \\
\hline
\end{tabular}

\section{DISCUSSION}

The SERPINE2 gene (GenBank accession No. NM_006216) contains nine exons, and encodes a 44-kDa cellular and extracellular matrix-associated serine protease inhibitor that is primarily involved in coagulation and fibrinolysis by inhibiting the activity of thrombin, urokinase, and plasmin (Baker et al., 1980; Scott et al., 1985). The expression of SERPINE2 is induced by interleukin- $1 \beta$, tumor necrosis factor- $\alpha$, and transforming growth factor- $\beta$, which have been shown to be involved in the development of COPD (Vaughan and Cunningham, 1993; Mbebi et al., 1999; Arja et al., 2014). While the role of SERPINE2 in COPD and emphysema has not yet been fully investigated, characterization of SERPINE2 polymorphisms through population-based studies may allow us to answer whether the product of SERPINE2 plays a major role in the pathogenesis of COPD.

The SNP in SERPINE2 included in this meta-analysis was rs3795879 G/A in intron 3. A number of published studies that focused on this polymorphism in the pathogenesis of COPD have made conflicting conclusions. Therefore, a meta-analysis was performed to systematically assess the possible association between the rs $3795879 \mathrm{G} / \mathrm{A}$ polymorphism in SERPINE2 and the development of COPD. To the best of our knowledge, this is the first study analyzing the association between the rs 3795879 polymorphism and COPD risk based on a meta-analysis. The results of the meta-analysis suggest no significant association between the rs3795879 G/A polymorphism and the risk of developing COPD. Moreover, analyzing the data based on the subgroups of race and the source of controls yielded the same result. However, significant heterogeneity was detected between all studies in the G vs A comparison for the rs3795879 polymorphism, whereas no heterogeneity was identified between the studies conducted with Asian populations. Interestingly, when the study by Demeo et al. (2006) was removed, the $\mathrm{I}^{2}$ of all studies in the $\mathrm{G}$ vs A comparison decreased from $59.3 \%(\mathrm{Ph}=0.04)$ to $0 \%(\mathrm{Ph}=0.551)$, while no significant association between the rs3795879 polymorphism and COPD risk was found either. Due to insufficient data on smoking status, which may affect the results of this meta-analysis, we did not take into account the potential interaction between genes and smoking in the present study. Thus, further studies based on data that includes individual and environmental factors such as smoking status, with unbiased genotyping methods and matched controls in different populations are necessary. In addition, the analysis of more SERPINE2 polymorphisms may allow us to draw more specific conclusions on the association between SERPINE2 and the risk of COPD.

There are a number of limitations in the present meta-analysis. First, the lack of the original data in some studies limited our evaluation on the potential effects of gene-gene and gene-environment interactions on the development of COPD. Second, the controls were not 
consistent among the published studies. Some studies employed a healthy population as the control group, whereas others used hospital patients without COPD as the controls. Thus, the controls may not always represent the underlying source populations. Lastly, these casecontrol studies were from Asia, Europe, and the United States and thus, the results may be applicable to those specific ethnicities.

In summary, the present meta-analysis suggests that the rs3795879 G/A polymorphism in SERPINE2 is not involved in the development of COPD. However, studies with larger sample size, detailed original data, standard genotyping methods, homogeneous COPD patients, and well-defined controls, are necessary to further investigate the role of SERPINE2 in the pathogenesis of COPD.

\section{Conflicts of interest}

The authors declare no conflict of interest.

\section{REFERENCES}

Arja C, Ravuri RR, Pulamaghatta VN, Surapaneni KM, et al. (2014). Genetic determinants of chronic obstructive pulmonary disease in South Indian male smokers. PLoS One 9: e89957.

Baker JB, Low DA, Simmer RL and Cunningham DD (1980). Protease-nexin: a cellular component that links thrombin and plasminogen activator and mediates their binding to cells. Cell 21:37-45.

Begg CB and Mazumdar M (1994). Operating characteristics of a rank correlation test for publication bias. Biometrics 50: $1088-1101$.

Carter RE, Cerosaletti KM, Burkin DJ, Fournier RE, et al. (1995). The gene for the serpin thrombin inhibitor (PI7), protease nexin $\mathrm{I}$, is located on human chromosome 2q33-q35 and on syntenic regions in the mouse and sheep genomes. Genomics 27: 196-199.

Chappell S, Daly L, Morgan K, Baranes TG, et al. (2006). The SERPINE2 gene and chronic obstructive pulmonary disease. Am. J. Hum. Genet. 79: 184-186.

Chen Y, Chen P, Hanaoka M, Droma Y, et al. (2008). Enhanced levels of prostaglandin E2 and matrix metalloproteinase-2 correlate with the severity of airflow limitation in stable COPD. Respirology 13: 1014-1021.

Decramer M, Janssens W and Miravitlles M (2012). Chronic obstructive pulmonary disease. Lancet 379: 1341-1351.

Demeo DL, Mariani TJ, Lange C, Srisuma S, et al. (2006). The SERPINE2 gene is associated with chronic obstructive pulmonary disease. Am. J. Hum. Genet. 78: 253-264.

Egger M, Davey SG, Schneider M and Minder C (1997). Bias in meta-analysis detected by a simple, graphical test. $B M J$ 315: 629-634.

Ezzati M and Lopez AD (2003). Estimates of global mortality attributable to smoking in 2000. Lancet 362: 847-852.

Fujimoto K, Ikeda S, Arai T, Tanaka N, et al. (2010). Polymorphism of SERPINE2 gene is associated with pulmonary emphysema in consecutive autopsy cases. BMC Med. Genet. 11: 159.

Haq I, Chappell S, Johnson SR, Lotya J, et al. (2010). Association of MMP-2 polymorphisms with severe and very severe COPD: a case control study of MMPs-1, 9 and 12 in a European population. BMC Med. Genet. 11: 7.

Higgins JP, Thompson SG, Deeks JJ and Altman DG (2003). Measuring inconsistency in meta-analyses. BMJ 327: 557-560.

Kabesch M and Adcock IM (2012). Epigenetics in asthma and COPD. Biochimie 94: 2231-2241.

Kukkonen MK, Tiili E, Hamalainen S, Vehmas T, et al. (2011). SERPINE2 haplotype as a risk factor for panlobular type of emphysema. BMC Med. Genet. 12: 157.

Li JZ, Absher DM, Tang H, Southwick AM, et al. (2008). Worldwide human relationships inferred from genome-wide patterns of variation. Science 319: 1100-1104.

Mbebi C, Hantai D, Jandrot-Perrus M, Doyennette MA, et al. (1999). Protease nexin I expression is up-regulated in human skeletal muscle by injury-related factors. J. Cell Physiol. 179: 305-314.

Petitti D (2000). Meta-analysis, decision analysis, and cost-effectiveness analysis: methods for quantitative synthesis in medicine. Oxford University Press, Cary.

Scott RW, Bergman BL, Bajpai A, Hersh RT, et al. (1985). Protease nexin. Properties and a modified purification procedure. J. Biol. Chem. 260: 7029-7034. 
Vaughan PJ and Cunningham DD (1993). Regulation of protease nexin-1 synthesis and secretion in cultured brain cells by injury-related factors. J. Biol. Chem. 268: 3720-3727.

Wang A, Yin Y, Chen P, Liu Q, et al. (2011). The association of SERPINE2 gene with COPD in a Chinese Han population. Yonsei Med. J. 52: 953-960.

Zhong L, Fu WP, Sun C, Dai LM, et al. (2009). Absence of association between SERPINE2 genetic polymorphisms and chronic obstructive pulmonary disease in Han Chinese: a case-control cohort study. BMC Med. Genet. 10: 66.

Zhu G, Warren L, Aponte J, Gulsvik A, et al. (2007). The SERPINE2 gene is associated with chronic obstructive pulmonary disease in two large populations. Am. J. Respir. Crit. Care Med. 176: 167-173. 